\title{
Efeito bifidogênico do frutooligossacarídeo na microbiota intestinal de pacientes com neoplasia hematológica
}

\author{
Bifidogenic effect of fructooligosaccharides in the \\ intestinal flora of patients with hematological neoplasia
}

Telma BÚRIGO1

Regina Lúcia Martins FAGUNDES²

Erasmo Benicio Santos de Moraes TRINDADE²

Helena Cristina Ferreira Franz VASCONCELOS ${ }^{3}$

\section{R E S U M O}

\section{Objetivo}

Verificar o efeito bifidogênico do frutooligossacarídeo nos pacientes com neoplasias hematológicas submetidos a quimioterapia.

\section{Métodos}

Trata-se de um estudo clínico randomizado duplo cego, desenvolvido na Unidade de Transplante de Medula Óssea do Centro de Pesquisas Oncológicas de Florianópolis, o qual envolve 25 pacientes divididos em 2 grupos que receberam, por 15 dias, $12 \mathrm{~g}$ de frutooligossacarídeo $(n=14)$ ou placebo (maltodextrina) $(n=11)$. Foram avaliados a quantidade de bifidobactérias e os valores de pH fecal antes e após a suplementação.

\section{Resultados}

Observou-se na população estudada o predomínio do sexo masculino (72\%) e a idade média de 34 anos. O grupo suplementado apresentou um aumento significante na quantidade de bifidobactérias $(p<0,05)$ e o $\mathrm{pH}$ fecal não foi alterado em nenhum dos grupos.

\section{Conclusão}

Verificou-se que a suplementação aumentou a quantidade de bifidobactérias, interferindo na composição da microbiota intestinal, e que não houve alteração do $\mathrm{pH}$ fecal.

Termos de indexação: bifidobactéria; microbiota; frutooligossacarídeos; neoplasias hematológicas.

\footnotetext{
1 Centro de Pesquisas Oncológicas. Florianópolis, SC, Brasil.

2 Universidade Federal de Santa Catarina, Centro de Ciências da Saúde, Programa de Pós-Graduação em Nutrição. Campus Universitário, Trindade, 88040-970, Florianópolis, SC, Brasil. Correspondência para/Correspondence to: R.L.M. FAGUNDES. E-mail: <remartin@ccs.ufsc.br>.

3 Universidade Federal de Santa Catarina, Centro de Ciências da Saúde, Curso de Graduação em Farmácia e Análises Clínicas, Departamento de Farmácia e Análises Clínicas. Florianópolis, SC, Brasil.
} 
492 | T. BÚRIGO et al.

\section{A B S T R A C T}

\section{Objective}

To verify the bifidogenic effect of fructooligosaccharides in patients with hematological neoplasia submitted to chemotherapy.

\section{Methods}

This is a clinical, randomized, double-blind study done in the Bone Marrow Transplant Unit of the Oncology Research Center of Florianopolis. It involved 25 patients divided into 2 groups who received $12 \mathrm{~g}$ of fructooligosaccharides ( $n=14$ ) for 15 days or placebo (maltodextrin) ( $n=11)$. The amount of bifidobacteria and the values of fecal $\mathrm{pH}$ before and after supplementation were investigated.

\section{Results}

Most of the studied population was male (72\%) and the mean age was 34 years. The group that received supplementation presented a significant increase in the amount of bifidobacteria $(p<0.05)$ and fecal $\mathrm{pH}$ remained unchanged in both groups.

\section{Conclusion}

Supplementation increased the amount of bifidobacteria, interfering in the composition of the intestinal flora, but fecal pH was not affected.

Indexing terms: bifidogenic microbiota; fructooligosaccharide; hematologic neoplasms.

\section{N T R O D U Ç Ã O}

O trato gastrointestinal (TGI) abriga uma flora de mais de 500 espécies de bactérias e sua população não está distribuída igualmente ao longo do sistema digestório. O cólon é o local que contém um complexo e dinâmico ecossistema microbiótico, com grande concentração de bactérias que chegam a atingir mais de $10^{11}$ a $10^{12}$ unidades formadoras de colônia por mililitro (UFC/mL) ${ }^{1-4}$.

O gênero bifidobactéria é o maior grupo de bactérias no cólon, o qual constitui mais de $25 \%$ do total da população intestinal adulta e 95\% em recém-nascidos. Promove diversos efeitos benéficos ao hospedeiro, tais como: fermentação de substratos, resultando na produção dos ácidos graxos de cadeia curta (AGCC); redução do $\mathrm{pH}$, que exerce ação bactericida; diminuiçãa dos níveis séricos de amônia pela fermentação de proteínas; participação na produção de vitaminas do complexo B e influência na resposta imune ${ }^{5-8}$.

As bifidobactérias possuem, ainda, a capacidade de exercer um efeito inibitório sobre o crescimento de outras espécies, o que leva a um menor risco de invasão e colonização por bactérias patogênicas para o organismo humano. Acredita- -se que esse processo de inibição utilizado por essas bactérias se dê pela produção de ácidos, principalmente do acetato e do lactato ${ }^{9-11}$.

O equilíbrio entre as espécies de bactérias residentes promove a estabilidade da população microbiana em condições normais. Entretanto, determinadas situações - como nas diarréias agudas, no tratamento antimicrobiano ou nas intervenções dietéticas restritivas - podem romper esse equilíbrio e permitir o crescimento excessivo das espécies com potencial patogênico, tais como o clostridium difficile, associado com colites pseudomembranosas ${ }^{12-14}$.

As neoplasias malignas hematológicas, que compreendem principalmente as leucemias, linfomas e mielomas, são caracterizadas por alterações no sistema imunológico que, em geral, são resultantes de uma combinação de fatores determinantes da própria doença, bem como do tratamento antineoplásico. A rigor, todos os componentes básicos da defesa do organismo podem ser afetados: a pele, as mucosas, a imunidade celular específica e inespecífica, bem como a imunidade humoral ${ }^{15}$.

A agressão à mucosa intestinal ocorre principalmente como conseqüência da terapia 
antineoplásica, visto que as células normais se multiplicam de forma semelhante às tumorais. A rapidez do turnover das células da mucosa do trato digestório as torna especialmente vulneráveis à quimioterapia, resultando em mucosites, ulcerações e diminuição da capacidade de absorção ${ }^{16}$.

O termo prebiótico foi introduzido por Gibson \& Roberfroid ${ }^{2}$ e definido como sendo um ingrediente alimentar não digerido, que resulta em beneficio ao hospedeiro pela estimulação seletiva do crescimento e/ou ativação do metabolismo de uma ou de um número limitado de bactérias no cólon.

Os frutooligossacarídeos (FOS) ou oligofrutoses são oligossacarídeos resistentes, isto é, carboidratos complexos de configuração molecular que os tornam resistentes à ação hidrolítica da enzima salivar e intestinal, atingindo intactos o cólon. O FOS é formado a partir da hidrólise da inulina pela enzima inulase e desempenha diversas funções fisiológicas no organismo, como a alteração do trânsito intestinal, promovendo: redução de metabólitos tóxicos; prevenção de câncer de cólon; redução do colesterol plasmático e da hipertrigliceridemia; melhora da biodisponibilidade de minerais, além de contribuir para o aumento da concentração das bifidobactérias no cólon ${ }^{17-20}$.

Diante da importância da microbiota intestinal para a saúde humana e considerando a inexistência de estudos aplicados à população oncológica, justifica-se a realização deste trabalho, com o objetivo de verificar o efeito do FOS sobre a concentração de bifidobactérias em indivíduos com neoplasias hematológicas submetidos a tratamento quimioterápico.

\section{MÉ T O D O S}

Este é um estudo do tipo ensaio clínico randomizado, duplo cego, de natureza quantitativa prospectiva. Foi desenvolvido na Unidade de Transplante de Medula Óssea (TMO) do Centro de Pesquisas Oncológicas (CEPON), localizado no Hospital Governador Celso Ramos (HGCR) em
Florianópolis, SC, durante o período de julho a novembro de 2005.

Foram estudados 25 pacientes com neoplasias hematológicas, submetidos ao tratamento com quimioterapia, na dose e/ou tempo de infusão de efeito imuno e mielosupressivo intenso, com tempo de internação igual ou superior a 15 dias. Foram considerados como critérios de inclusão os pacientes que apresentaram os seguintes diagnósticos: leucemia mielóide aguda (LMA), leucemia linfóide aguda (LLA), linfoma de Hodgkin (LH), linfoma não Hodgkin (LNH), e pacientes que aceitaram alimentação por via oral.

O estudo foi submetido e aprovado pelo Comitê de Ética em Pesquisa do CEPON, tendo sido obtido o Termo de Consentimento Livre e Esclarecido de todos os participantes.

Os pacientes foram distribuídos aleatoriamente em dois grupos por sorteio, constituindo-se em "grupo controle" com 11 pacientes (G1), que receberam suplementação de placebo (maltodextrina), e "grupo suplementado" com 14 pacientes (G2), que receberam suplementação de FOS. A maltodextrina e o FOS foram doados devidamente embalados e identificados como A e B, sendo administrados diariamente por um período de 15 dias a partir do primeiro dia da quimioterapia (dia 1), da seguinte forma: 1 envelope de $6 \mathrm{~g}$ diluídos em $100 \mathrm{~mL}$ de água mineral, 2 vezes ao dia, totalizando $12 \mathrm{~g} / \mathrm{dia}$, via oral. Foram coletados dados de sexo, diagnóstico e idade de todos os participantes do estudo.

Para o isolamento e a quantificação de bifidobactérias e do pH fecal, amostras de fezes foram coletadas na unidade de TMO, antes e após a suplementação, em sacos plásticos individuais com fecho hermético; tais amostras foram analisadas no Laboratório de Microbiologia Clínica do Departamento de Análises Clínicas da Universidade Federal de Santa Catarina (UFSC).

As verificações de $\mathrm{pH}$ das amostras foram realizadas na diluição $0,1 \mathrm{~mL}$, utilizando fitas de pH Quimilabor ${ }^{\circledast}$ (3 tipos de fitas com diferentes faixas de $\mathrm{pH}: 2.5-4.5 / 4.0-7.0 / 6.5-10.0)^{19}$. 
A quantificação de bifidobactérias foi determinada de acordo com o protocolo estabelecido pelo Instituto de Tecnologia em Alimentos (ITAL) (Campinas, SP), publicado por Gibson \& Wang ${ }^{13}$ e por Muñoa \& Pares $^{21}$, conforme descrito a seguir: as amostras fecais dos pacientes selecionados foram mantidas em ambiente anaeróbio por, no máximo, 8 horas até o momento da análise. 0 meio seletivo BIM-25: RCA (Reinforced Clostridial agar $\left.{ }^{\circledR}\right)$ foi adicionado de alguns antibióticos, havendo sido utilizado um tampão fosfato para diluição e autoclavado a $121^{\circ} \mathrm{C}$ durante 15 minutos, e estocado em geladeira. Foram preparados frascos para as diluições decimais seriadas até $10^{-7}$. As placas corretamente identificadas com o nome do paciente e as respectivas diluições foram incubadas a $37^{\circ} \mathrm{C}$ durante 72 horas em jarra anaeróbia, tendo sido utilizado o sistema comercial de geração de atmosfera anaeróbia (Anaerobac da Probac $\left.{ }^{\circledR}\right)$. Após o período de incubação, realizou-se a contagem das colônias de bifidobactérias, sendo que o resultado foi apresentado em escala logarítmica (log) de unidade formadora de colônia por mililitro de fezes (UFC/mL).

Foi observado o crescimento de, no máximo, quatro diferentes tipos de colônias nas placas, para todos os pacientes estudados (colônias brancas, cor-de-rosa claras, cor-de-rosa escuras e de cor púrpura), tendo sido feita a coloração de Gram para todos os tipos de colônias. A microscopia mostrou em todas elas a morfologia esperada e a característica das bifidobactérias, ou seja, a presença de bacilos Gram-positivos curtos, algumas vezes cocóides, arranjados isoladamente, aos pares, em cadeias e/ou com ramificações ${ }^{13,21}$.

Para a análise estatística, os dados foram organizados e registrados no programa Exce ${ }^{\circledR} 2000$ e inseridos no programa estatístico Statistical Package for the Social Sciences (SPSS), versão 10.0 para o Windows. Para análise das variáveis, foi utilizado o teste ANOVA multivariada de duas vias, considerando-se a interação dos grupos (controle e suplementado) e o tempo (inicial e final). Os dados também foram analisados pela diferença (final - inicial) entre as variáveis, utili- zando-se o teste " $t$ " - Student não pareado. Escolheu-se a análise pelas diferenças com vistas a reduzir a variabilidade individual da amostra. Verificou-se que a quantidade de bifidobactérias não apresentava distribuição normal e, neste caso, utilizou-se o teste não-paramétrico de Mann-Whitney.

As variáveis quantitativas, como idade, $\mathrm{pH}$ e bifidobactérias, foram apresentadas em média e acompanhadas do desvio-padrão (DP) na análise descritiva. Já as variáveis nominais, como diagnóstico e sexo, foram descritas em categorias de freqüência a partir do aparecimento nos grupos descritos, utilizando-se o teste qui-quadrado para comparação das suas diferenças. Para todos os testes, adotou-se o nível de significância de 95\% $(p<0,05)$.

\section{RES U LTA D OS}

De acordo com a caracterização da amostra, observa-se que, quanto à faixa etária (Tabela 1), as médias de idade são semelhantes nos grupos controle e suplementado, assim como a distribuição por sexo e por diagnóstico, sem diferença estatística. No entanto, quando se analisa a distribuição dos pacientes estudados quanto ao sexo, observa-se predomínio do sexo masculino, com $72 \%$, contra $28 \%$ para o feminino. Quanto ao tipo de neoplasia, houve maior freqüência dos casos de leucemias, representando $60 \%$, enquanto a freqüência dos linfomas foi de $40 \%$.

Tabela 1. Distribuição de pacientes com neoplasias hematológicas, quanto ao sexo, idade e diagnóstico, em ensaio clínico. Florianópolis, 2005.

\begin{tabular}{lcc}
\hline Variável & Grupo controle $(n=11)$ & Grupo suplementado $(n=14)$ \\
\hline Sexo & 9 & 9 \\
Masculino (n) & 2 & 5 \\
Feminino (n) & 34,45 DP 14,54 & 33,93 DP 12,28 \\
Idade (anos)* & $(18-60)$ & \\
Diagnóstico & & 9 \\
Leucemias & 6 & 5 \\
Linfomas & 5 & \\
\hline
\end{tabular}

*Valores expressos em média e desvio-padrão (DP) 
Quanto aos dados referentes à quantidade de bifidobactérias (Figura 1), em escala logarítmica (log) nos grupos estudados, verificou-se que houve diferença significante entre os grupos em relação ao tempo e suplemento $(U=45,000 ; p=0,045)$. 0 grupo suplementado apresentou crescimento do conteúdo de bifidobactérias no final do período ( $\log 11,8 \mathrm{DP}=1,5$ para $\log 14,8 \mathrm{DP}=1,3$ ) enquanto

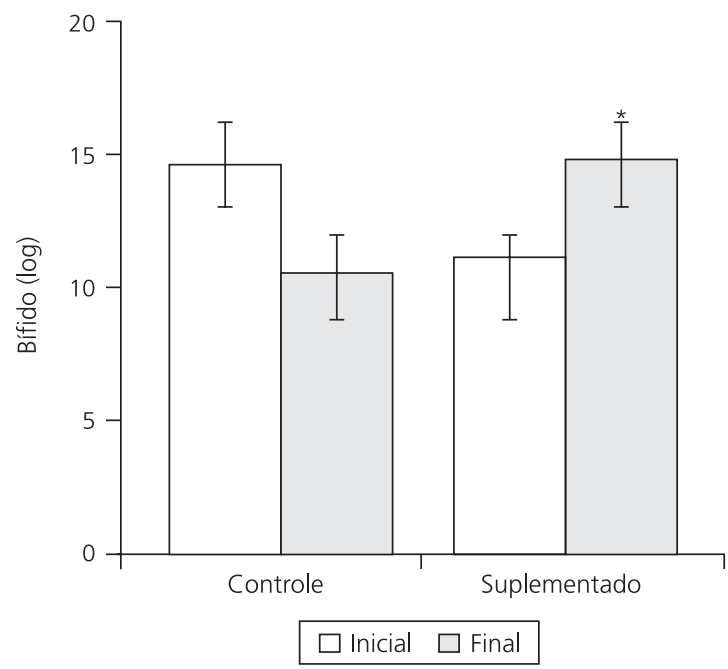

Figura 1. Quantidade (média e desvio-padrão) de bifidobactérias em log, em ensaio clínico de pacientes com neoplasias hematológicas, e suas diferenças na interação entre grupo e tempo. Florianópolis, 2005.

Legenda: Dados representados pela média e desvio-padrão; ${ }^{*} p<0,05$.

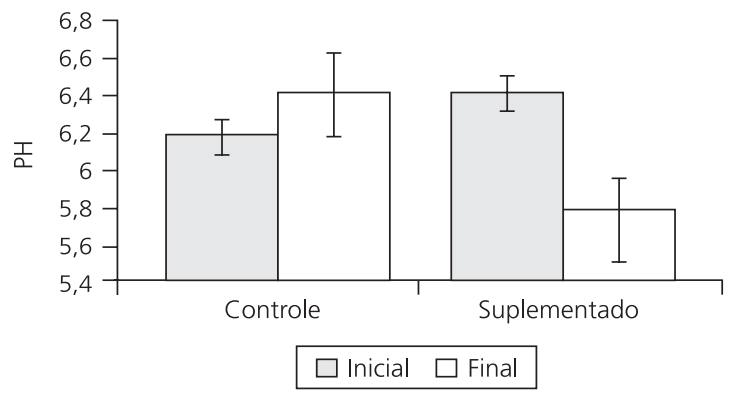

Figura 2. Valores de $\mathrm{pH}$ em ensaio clínico de pacientes com neoplasias hematológicas e a suas diferenças na interação entre grupo e tempo de estudo. Florianópolis, 2005.

Legenda: dados representados pela média e desvio-padrão; $p=0,095$. no grupo controle, houve uma redução do conteúdo de bifidobactérias ( $\log 14,5 \mathrm{DP}=1,7$ para $\log 10,6 \mathrm{DP}=1,1)$.

No que diz respeito ao $\mathrm{pH}$ fecal, não houve diferença significante entre os grupos após uso do FOS. O pH manteve-se estável, em torno de $6,4, \mathrm{DP}=0,6$ e 5,8, $\mathrm{DP}=0,5(p=0,095)$, nos grupos controle e suplementado, respectivamente (Figura 2), porém pode-se notar uma pequena redução do $\mathrm{pH}$ no grupo suplementado e uma discreta elevação no grupo controle.

\section{I S C U S S Ã O}

Os indivíduos deste estudo estavam submetidos a diversas situações de estresse, como alteração do consumo alimentar devido à internação hospitalar, ação de drogas citotóxicas que compõem os protocolos de tratamento e seus efeitos colaterais, além da ação direta da doença de base, agravando ainda mais seu estado nutricional e alterando a microbiota bacteriana intestinal normal.

As neoplasias malignas hematológicas ocupam o quinto lugar na incidência de câncer, segundo dados do Instituto Nacional do Câncer (INCA), sendo que o tipo de neoplasia mais freqüente é a leucemia, que representa, aproximadamente, $10,7 \%$ dos casos registrados no País, sendo predominante em indivíduos do sexo masculino.

Com relação à caracterização da amostra no presente trabalho, observa-se que a maioria dos pacientes é do sexo masculino, apresentando perfil semelhante aos dados oficiais e encontrados por Ellis et al. ${ }^{22}$. O mesmo ocorreu em relação à idade e ao tipo de neoplasia, visto que a idade média observada foi de adultos jovens com predomínio de portadores de leucemias (60\%).

Ao analisar o conteúdo de bifidobactérias na população deste estudo, observou-se efeito positivo do uso do prebiótico FOS no grupo suplementado, no qual houve um aumento significante do conteúdo de bifidobactéria. No estudo de 
Gibson \& Roberfroid², 8 indivíduos saudáveis receberam $15 \mathrm{~g}$ de FOS por um período de 15 dias; verificou-se que a suplementação de FOS nesses indivíduos saudáveis promoveu o aumento significativo das bifidobactérias, enquanto os bacteróides, fusobactérias e clostrídio foram reduzidos, sugerindo que a dieta pode alterar a microbiota colônica.

O trabalho desenvolvido por Bouhnik et al. ${ }^{23}$, utilizando $10 \mathrm{~g}$ diárias de transgalacto-oligossacarídeo (prebiótico similar ao FOS) em indivíduos saudáveis e em amostra in vitro, mostrou o aumento do número de bifidobactérias em ambas as situações. Já Kleessen et al. ${ }^{24}$ observaram que doses gradativas, a partir de $20 \mathrm{~g}$ de inulina até $40 \mathrm{~g}$ por 19 dias, em mulheres idosas, aumentaram significativamente de 7,9 para 9,2 $\log _{10} / \mathrm{g}$ a quantidade de bifidobactérias fecais, com diminuição de enterococcus e enterobactérias.

Neste trabalho, quando se avaliaram os valores de $\mathrm{pH}$ na população estudada, verificou-se que não houve diferença estatisticamente significante entre os grupos $(p=0,095)$. De acordo com a literatura, a fermentação resulta em diminuição do pH colônico; baixos valores de $\mathrm{pH}$ impedem o crescimento de certas espécies de bactérias patogênicas enquanto favorecem o crescimento das espécies benéficas ${ }^{25}$.

Entretanto, o resultado encontrado no presente trabalho sugere que a suplementação do FOS pode ter favorecido o crescimento somente de algumas espécies de bifidobactérias nesta população. Essa hipótese foi testada por Garro et al. ${ }^{26}$, que verificaram a influência do $\mathrm{pH}$ na atividade das bifidobactérias. Foi avaliado o crescimento da bifidobactéria da espécie longum em um estudo in vitro com o consumo de rafinose (prebiótico similar ao FOS). Este trabalho mostrou que nos pHs 6,0; 5,5 e 5,0 o substrato foi completamente consumido. Já em pH 4,5 o crescimento foi menor.

Cumpre salientar que, em outro trabalho realizado por Klessen et al. ${ }^{24}$ para avaliar o efeito da suplementação de inulina e lactose em idosos, não foram observadas alterações no pH fecal, que permaneceu em valores constantes entre 7,3, $D P=0,6$ e 7,5, DP=0,6. Segundo esses autores, para que o metabolismo e o conteúdo intestinal possam ser analisados nas fezes, fatores que ocorrem no cólon, tais como a mobilidade intestinal, o consumo total de fibra ingerida na dieta, as secreções intestinais, como a produção de ácidos graxos de cadeia curta, e a duração da intervenção dietética deverão ser considerados. Nesse sentido, o pH fecal pode não refletir com exatidão o pH no cólon.

Concluiu-se, portanto, que a suplementação do prebiótico frutooligossacarídeo (FOS) foi capaz de aumentar a quantidade de bifidobactérias e não promoveu a diminuição do pH fecal.

\section{A GRADECIMENTOS}

Ao Programa de Pós-graduação em Nutrição da Universidade Federal de Santa Catarina, ao Departamento de Análises Clínicas/UFSC e ao Centro de Pesquisas Oncológicas.

\section{COLABORADORES}

T. BÚRIGO participou na concepção e desenho da pesquisa, coleta de dados, análise e interpretação dos dados e redação do trabalho. R.L.M. FAGUNDES orientou a concepção e o desenho metodológico, a análise e a interpretação dos dados e fez a revisão final do manuscrito. E.B.S.M. TRINDADE colaborou na análise, interpretação dos dados e redação do manuscrito. H.C.F.F. VASCONCELOS responsável pelas análises microbiológicas de pH e determinação de bifidobactérias.

\section{REFER Ê NCIAS}

1. Bourlioux P, Koletzko B, Guarner F, Braesco V. The intestine and its microflora are partners for the protection of the host: report on the Danone Symposium "The intelligent intestine", held in Paris. Am J Clin Nutr. 2003; 78: 675-83. 
2. Gibson GR, Roberfroid MB. Dietary modulation of human colonic microbiota: introducing the concept of the prebiotics. J Nutr. 1995; 125 : 1401-12.

3. Berg R. The indigenous gastrointestinal microflora. Trends Microbiol. 1996; 4(11):430-5.

4. Bengmark S. Ecological control of the gastrointestinal tract. The role of probiotic flora. Gut. 1998; 42:2-7.

5. Blaut, M. Relationship of probiotics and food to intestinal microflora. Eur J Nutr. 2002; 41(Suppl 1):11-6.

6. Bengmark S. Immunonutrition: role of biosurfactants fiber and probiotic bacteria. Nutrition. 1998; 14(7/8):585-94.

7. Guarner F. El colon como órgano: hábitat de flora bacteriana. Nutr Hosp. 2002; (Suppl 2):2-7.

8. Peris GP, Lesmes B, Cuerda Compes CM, Álvarez C. Metabolismo colônico de la fibra. Nutr Hosp. 2002; 17:11-6.

9. Leahy SC, Higgins DG, Fitzgerald, Sinderen D van. A review: getting better with bifidobacterium. J Appl Microbiol. 2005; 98:1303-15.

10. Ishibashi N, Yaeshima T, Hayasawa $H$. Bifidobacteria: their significance in human intestinal health. Mal J Nutr. 1997; 3:149-59.

11. Guarner F, Malagelada JR. Gut flora in health and disease. The Lancet. 2003; 361(8):512-9.

12. Hopkins MJ, MacFarlane GT. Nondisgestible oligosaccharides enhance bacterial colonization resistance against Clostridium difficile in vitro. Appl Env Microbiol. 2003; 69(4):1920-7.

13. Gibson GR, Wang X. Regulatory effects of bifidobacteria on the growth of the colonic bacteria. J Appl Bacteriol. 1994; 77(4):412-20.

14. Gibson GR. Dietary modulation of the human gut microflora using the prebiotics oligofructose and inulin. J Nutr. 1999; 129:1438-41s.

15. Nucci M. Infecções no paciente com neoplasia hematológica: diagnóstico, tratamento e prevenção. In: Zago MA, Falcão RP, Pasquini R. Hematologia: fundamentos e prática. São Paulo: Atheneu; 2001. cap.44:419-29.
16. Daly JM, Hoffman K, Lieberman M, Leon P, Redmond HP, Shou J, et al. Nutritional support in the cancer patient. JPEN. 1990; 14(5):244-8s.

17. Watzl B, Girrbacch S, Roller M. Inulin, oligofructose and immunomodulation. Br J Nutr. 2005; 93(Suppl 1): 49-55.

18. Cummings JH, Macfarlane GT, Englist HN. Prebiotics digestion and fermentation. Am J Clin Nutr. 2001; 73(Suppl):415-20.

19. Bengmark S, Lorenzo AG, Culebras MJ. Use of pro, pre and symbiotics in the ICU-future options. Nutr Hosp. 2001; 16(6):239-56.

20. Cummings JH, MacFarlane GT. Gastrointestinal effects of prebiotics. Br J Nutr. 2002; 87(Suppl 2):145-51

21. Muñoa FJ, Pares R. Selective medium for isolation and enumeration of bifidobacterium spp. Appl Envl Microbiol. 1988; 54(7):1715-8.

22. Ellis M, Zwaan F, Hedström U, Poynton C, Kristensen J, Jumaa $P$, et al. Recombinant human interleukin 11 and bacterial infection in patient with haematological malignant disease undergoing chemotherapy: a double-blind placebo-controlled randomized trial. Lancet. 2003; 361(25):275-80.

23. Bouhnik $Y$, Flourié $B$, Abensour DA, Pochart $P$, Gramet G, Durand $M$, et al. Administration transgalacto-oligosaccharides increases fecal bifidobactéria and modifies colonic fermentation metabolism in healthy humans. J Nutr. 1997; 127 : 444-8.

24. Kleessen B, Sykura B, Zunfit HJ, Blaut M. Effect of inulin and lactose on fecal microflora, microbial activity, and bowel habit in elderly constipated persons. Am J Clin Nutr. 1997; 65:1397-02.

25. Gibson GR. Fiber and effect on prebiotics (The prebiotic concept). Clin Nutr Suppl. 2004; 1:25-31.

26. Garro MS, Aguirre L, Giori GS. Biological activity of bifidobacterium longum in response to environmental $\mathrm{pH}$. Appl Microbiol Biotechnol. 2005; (7):92-7.

Recebido em: 27/4/2006

Versão final reapresentada em: 20/7/2007 Aprovado em: 21/8/2007 
